International Journal of Algebra, Vol. 8, 2014, no. 4, 181 - 186

HIKARI Ltd, www.m-hikari.com

http://dx.doi.org/10.12988/ija.2014.3877

\title{
Countable Dimensional Right Perfect Ring
}

\author{
R. H. Sallam \\ Mathematics Department, Faculty of Science \\ Helwan University, Cairo, Egypt
}

Copyright (C) 2014 R. H. Sallam. This is an open access article distributed under the Creative Commons Attribution License, which permits unrestricted use, distribution, and reproduction in any medium, provided the original work is properly cited.

\begin{abstract}
We prove that a right self right perfect algebra which is at most countable dimensional modulo their Jacobson radical is right artinian.
\end{abstract}

Mathematics Subject Classification: 16D50, 16L60

Keywords: self injective rings, right perfect rings, quasi-frobenius rings

\section{Introduction}

In this note we present a proof for algebra $\mathrm{R}$ over a field $\mathrm{K}$ which is at most countable dimensional modulo their Jacobson radical i.e. $R / \operatorname{Rad}(\mathrm{R})$ is at most $\mathrm{N}_{0}$ This includes for example the important situation when R/ RadR is not only semisimple but also finite dimensional'

Let $\mathrm{R}$ be a ring with identity and RadR is its Jacobson radical. If $\mathrm{R}$ is right perfect (i.e. $\mathrm{R} / \mathrm{RadR}$ is semisimple and RadR is left T-nilpotent) then 
$\mathrm{R} / \mathrm{RadR}$ is semisimple and hence $(\mathrm{R} / \mathrm{RadR})_{\mathrm{R}}$ and ${ }_{\mathrm{R}}(\mathrm{R} / \mathrm{RadR})$ are semisimple right and left R-module.

Note that since $\mathrm{R} / \mathrm{RadR}$ is semisimple then an R-module $\mathrm{M}$ is semisimple iff it is cancelled by RadR. Indeed every simple is cancelled by $\operatorname{RadR}$ and if $\operatorname{RadM}=0$ then $\mathrm{M}$ has $\mathrm{R} / \mathrm{RadR}$-module structure which is semisimple ,therefore $\mathrm{M}$ is semisimple as the lattice of R-submodules and R/RadR sub-modules coincide in this case.

$(\mathrm{R} / \mathrm{RadR})_{\mathrm{R}}$ is semisimple and finitely generated, so it has a composition series

$$
\text { i.e. } \quad J / J^{2} \subset J^{2} / J^{3} \subset \ldots \subset J^{n-1} / J^{n}
$$

is of length n-1 where each $J^{k} / J^{k+1}$ is semisimple for all $\mathrm{k}$.

Note that if $\mathrm{R}$ is right self injective then each decomposable $(\mathrm{eR})$ has simple socle.

Indeed if we have a nontrivial decomposition of the $\operatorname{socle}(e R)=M \oplus N$

Then we can find $\mathrm{E}(\mathrm{M})$ and $\mathrm{E}(\mathrm{N})$ injective hulls of $\mathrm{M}, \mathrm{N}$ contained in $\mathrm{eR}$ and we obtain

$\mathrm{eR}=\mathrm{E}(\mathrm{M})+\mathrm{E}(\mathrm{N})$ nontrivial decomposition that is a contradiction.

Note also that if $\mathrm{R}$ is right self injective right perfect then for each simple

Right R-module, the left module $\operatorname{Hom}(\mathrm{S}, \mathrm{R})$ is simple.

First Hom ( $\mathrm{S}, \mathrm{R})$ is non zero. Looking for the isomorphism types of indecomposable modules eR, these are projective, local and the cover of some simple R-module.

The number of isomorphism types of such modules equals the number of isomorphism types of simple modules equal $t$, say.

Moreover since the indecomposable eR's also injective with simple socle we see that they are isomorphic if and only if their socle isomorphic. This shows that the distinct types of isomorphism of simples occurring as socle of some $\mathrm{eR}$ is also $\mathrm{t}$, and so each simple $\mathrm{S}$ must appears as a socle of some $\mathrm{eR}$ (i.e. it embeds in $\mathrm{R}$ ) this shows that , for each simple R-modules S. If $f, g \in \operatorname{Hom}(S, R)$ and $\operatorname{Hom}(S, R) \neq 0$

$$
f: S \rightarrow R f \neq 0, \text { then }
$$

Is a monomorphism and since $\mathrm{R}_{\mathrm{R}}$ is injective then there is some $h: R \rightarrow R$ Such that $h \circ f=g$.If $f(x)=x c \quad \forall x \in R$ we get $f(x) . c=g(x)$ i.e. $f . c=g$ in $\operatorname{Hom}(S, R)$, this shows that $\operatorname{Hom}(S, R)$ is generated by $f \neq 0$ so it is simple.

In particular since each simple module embeds in $\mathrm{R}$ which is right self injective, it follows that $\mathrm{R}$ is injective cogenerator in the category of right $\mathrm{R}$-module, i.e. it is a right PF (Pseudo- Frobonius) ring. 


\section{Main Results}

Let $\mathrm{s}$ be a set of representatives' for simple right $\mathrm{R}$-modules and let $\mathrm{t}=|\mathrm{s}|$ and $R / \operatorname{RadR}=\oplus S^{K_{s}}$. Let $\mathrm{W}=$ socle $\left(\mathrm{R}_{\mathrm{R}}\right)$ be the right socle of $\mathrm{R}$ so $\mathrm{W}$ is an $\mathrm{R}$-subbimodule of $\mathrm{R}$.

Since each indecomposable module ( $\mathrm{Re}$ ) has simple socle, we have

Length (W) equals the number of terms in the indecomposable decomposition $R=\underset{S}{\oplus} \operatorname{Re}$ which equals length $(\mathrm{R} / \mathrm{RadR})_{\mathrm{R}}$, since each

Indecomposable $(\mathrm{Re})$ is local

Let $W=\oplus S^{P_{s}}$ we have $\sum_{S \in s} P_{s}=\sum_{S \in s} K_{s}$

\section{Proposition 1}

Let $\mathrm{R}$ be a right self injective right perfect ring then the set $\{\operatorname{Hom}(S, R) ; S \in s\}$ is a set of representatives for simple left R-modules.

In particular $\operatorname{Hom}(S, R)$ and $\operatorname{Hom}(T, R)$ are nonisomorphic for nonisomorphic,

$S, T \in R$

Proof

Since $\mathrm{R}$ is right self injective the monomorphism

$0 \rightarrow W \rightarrow R \quad, \quad$ gives rise to the epimorphism

Hom $(R, R) \rightarrow$ Hom $\quad(W . R) \rightarrow \quad 0$

Note that

Hom $(W, R)=\oplus \operatorname{Hom}(S, R)$

Since Hom $(S, R) \neq 0 \forall S \in S$, we have

Hom $(W, R)=\oplus \operatorname{Hom}(S, R)^{P_{s}}$,

has length equal to the length $W=\sum_{S \in S} P^{s}=\operatorname{length}(R / R a d R)_{R}$

By classical Wedderburn -Artin Theorem:

length $(R / \operatorname{RadR})_{R}=$ length ${ }_{R}(R / \operatorname{Rad} R)$

Since $\operatorname{Hom}(W, R)$ is semisimple, the kernel of $\quad R \rightarrow \operatorname{Hom}(W, R)$

Contains $R a d R$ and therefore since

length ${ }_{R}(R / \operatorname{Rad} R)=$ length $\operatorname{Hom}_{R}(W, R)$,

we obtain $R / R a d R \cong \operatorname{Hom}(W, R)$ as left R-module .

This shows that all types of isomorphism of simple left R-modules are found among components of $\operatorname{Hom}(W, R)$ and the statement is proved 
Note: we note that the above proof further shows that there is an exact sequence of left R-modules

$$
0 \rightarrow \operatorname{Rad} R \rightarrow R \rightarrow \operatorname{Hom}(W, R) \rightarrow 0
$$

This means that

$$
\{r \in R \text {, suchthat } \quad, r W=0\}=\operatorname{RadR} \quad \text {. That is } \operatorname{ann}\left({ }_{R} W\right)=\operatorname{Rad} R
$$

This shows that $\mathrm{W}$ is also semisimple as a left $\mathrm{R}$-module , i.e. the right socle of $\mathrm{R}$ is contained in the left socle, and hence the left and the right socle or the right PF ring coincide.

For a right $\mathrm{R}$-module $\mathrm{M}$, denote $M^{*}=\operatorname{Hom}(M, R)$ this is a left R-module.

\section{Proposition 2}

Let $\mathrm{R}$ be a right injective ring and let $\mathrm{M}$ be a right $\mathrm{R}$-module such that there is an exact sequence

$$
0 \rightarrow S_{R} \rightarrow M_{R} \rightarrow L_{R}^{(\alpha)} \rightarrow 0
$$

With $\mathrm{S}$, L simple modules and assume $\mathrm{S}=$ socle $(\mathrm{M}), L_{R}{ }^{(\alpha)}$ denote the coproduct of $\alpha$ copies of $\mathrm{L}_{\mathrm{R}}$. Then $M^{*}$ is local left $\mathrm{R}$-module with unique maximal ideal

$$
S^{\perp}=\left\{f \in \text { Hom }(M, R) ; f \mathrm{I}_{S} \neq 0\right\}
$$

Which is semisimple isomorphic to $\left(L_{R}{ }^{*}\right)^{(\alpha)}$

\section{Proof}

Since

$$
0 \rightarrow S_{R} \rightarrow M_{R} \rightarrow L_{R}^{(\alpha)} \rightarrow 0
$$

is an exact sequence, so we get the exact sequence,

$$
0 \rightarrow \operatorname{Hom}\left(L^{(\alpha)}, R\right) \rightarrow \operatorname{Hom}(M, R) \rightarrow \operatorname{Hom}(S, R) \rightarrow 0
$$

That is

$$
0 \rightarrow\left(L^{*}\right)^{\alpha} \rightarrow M^{*} \rightarrow S^{*} \rightarrow 0
$$

The kernel of the morphism

$$
\begin{aligned}
& M^{*}=\operatorname{Hom} \quad(M, R)-\frac{\gamma^{*}}{} \rightarrow S^{*}=\operatorname{Hom}(S, R) \\
& \text { Is } \quad\left\{f \in \operatorname{Hom} \quad(M, R) ; f \mathrm{I}_{S} \neq 0\right\}=S^{\perp}
\end{aligned}
$$

Hence $S^{\perp} \cong\left(L^{*}\right)^{\alpha}$ which is left semisimple module since it is cancelled by RadR.

Now since $\mathrm{M}$ has simple socle, and its socle embeds in $\mathrm{R}$ which is injective it follows that $\mathrm{M}$ embeds in $\mathrm{R}$. We note that $M^{*}$ is generated by any $f \notin S^{\perp}$ which will show 
that $M^{*}$ is cyclic. Indeed such $f$ must be a monomorphism and given any other $h: M \rightarrow R$.

By injectivity of $R_{R}$ there is $g \in \operatorname{Hom}(R, R)$ such that $g \circ f=h$. If $g(x)=c x$

for $c \in R$ then we have $h=c . f$ in $M^{*}$. This shows that $f . . R=M^{*}$ which shows that $S^{\perp}$ is the only maximal submodules of the cyclic left R-module $M^{*}$, that ends of the proof.

Note that the fact that $M^{*}$ is local can also be proved by embedding $\mathrm{M}$ in some indecomposable (eR) for an indecomposable idempotent e, and then, by applying the exact functor $\operatorname{Hom}(-, R)$ one obtain an epimorphism

Hom $(e R, R)=e R \rightarrow M^{*}$, and so $M^{*}$ is local because $e R$ is.

Let $\alpha$ be the largest cardinality for which there is a right $\mathrm{R}$-module $\mathrm{M}$ with simple socle and such that $M /(\operatorname{socle}(M)) \cong L^{\alpha}$ for some simple module $\mathrm{L}$.

Such cardinality exists, since any such module is contained in $\mathrm{R}$ because $\mathrm{R}$ is injective. In fact if $W_{1}$ is the second socle of $\mathrm{R}$, then $\alpha \leq$ length $\left(W_{2} / W\right)$. we note that if $\alpha$ is infinite this is an equality .

Indeed if for each simple modules $\mathrm{S}, \mathrm{L}$ we denote by $\alpha_{S, L}=[(E(S) / S): L]$-the multiplicity of $L$ in the second socle of the injective hull $\mathrm{E}(\mathrm{S})$ of $\mathrm{S}$, then $\quad \alpha=\max _{S, L}\left(\alpha_{S, L}\right)$.

Therefore $\alpha \leq \sum_{S, L \in S} \alpha_{S, L} \leq n \alpha=\alpha$ if $\alpha$ is infinite.

We note also that if $\sum_{k}$ is the $\mathrm{k}^{\text {th }}$ socle then the length $\quad\left(W_{k} / W_{k-1}\right) \leq \alpha \quad$; this follows by induction on $\mathrm{k}$.

If this is true for $\mathrm{k}$, then there is an embedding

$W_{k} / W_{k-1} \hookrightarrow R^{(\alpha)}$

And therefore we have

length $\left(W_{k+1} / W_{k}\right) \leq$ length $\left(W_{1} / W_{0}\right)^{(\alpha)}=\alpha \times \alpha=\alpha$, since $\alpha$ is infinite cardinal. So we have the following theorem:

\section{Theorem}

Let $\mathrm{R}$ be a right self injective right perfect algebra such that the dimension of each simple R-module is at most countable (equivalently the dimension of $R / R a d R$ is at most countable) hence $\mathrm{R}$ is countable, and hence right artinian 


\section{Proof}

With the above notation, assume $\alpha$ is infinite. The length of each $W_{k} / W_{k-1}$ is at most $\alpha$, so since the dimension of each simple is at most $\boldsymbol{\aleph}_{0}$, its dimension is at most $\boldsymbol{\aleph}_{0}, \times$ $\alpha=\alpha$ (since $\alpha$ is infinite).

Thus the dimension of $\mathrm{R}$ is at most $\alpha$, and so it equals $\alpha$ (since length $\left(W_{1} / W_{0}\right)=\alpha$ ).

On the other hand, by proposition (2.2) there is a local left R-module $M^{*}$, with socle $L^{\alpha}$ for some simple $L$.

Note that $\operatorname{dim}\left(L^{\alpha}\right) \geq 2^{\alpha}$ and that there is an epimorphism $R \rightarrow M^{*}$

So $\operatorname{dim}(R) \geq 2^{\alpha}$, this is a contradiction.

By the result of Lawrence [3] $\mathrm{R}$ is right self injective countably generated algebra over a field so it is quasi -frobenius and hence right artinian.

\section{References}

[1] F.W. Anderson and K. R. Fuller, Rings and Categories of Modules SpringerVerlag, Heidelberg, New York, (1991).

[2] F. Kasch, Modules and Rings, London Mathematical Society Monographs" Vol.17, Academic Press, New York, (1982).

[3] J. Lawrence, a countable self injective ring is quasi-frobenius, proceeding of the American mathematical society, volume65, No.2, august 1977.

[4] M.C.Iovanov, semiprimary selfinjective algebras with at most countable dimensional Jacobson quotient are quasi frobenius, arxiv:1111.2901, 2011.

[5] T-Y. Lam, lectures on modules and rings, Graduate Texts in Mathematics, No.189. Berlin-New York, Springer-Verlag (1999).

\section{Received: August 1, 2014}

